\title{
The use of the EEG in clinical psychiatry
}

\author{
P. C. W. BowIE, Consultant Psychiatrist, High Royds Hospital, and Honorary Clinical \\ Lecturer, Department of Psychiatry, University of Leeds; A. Y. BEAINI, Consultant \\ Psychiatrist, Scalebor Park Hospital, and Honorary Lecturer, Department of \\ Psychiatry, University of Leeds; and A. A. DA CosTA, Consultant Neurophysiologist, \\ Department of Neurophysiology, St James's Hospital, Leeds 9
}

The electroencephalogram (EEG) is widely reported to be of limited value in psychiatry. This is particularly so with regard to the neuroses and functional psychoses. The value of the EEG in organic states is not much better, especially since the advent of computerised tomography (CT). However, it should be noted that the former gives a functional and the latter a structural assessment of cerebral disturbance.

Apart from standard recording and qualitative analysis, the relationships between psychiatric diagnosis and evoked brain potentials and quantitative EEG variables have been extensively studied but, again, with limited results. This is particularly so with regard to the quantitative EEG variables from which diagnostic groups can be classed according to the level of EEG activation, but there is no indication that any diagnostic group can be uniquely distinguished from any other by means of this EEG technique.

The high incidence of EEG abnormalities in criminals has led to numerous diagnosis related investigations. The psychopathic personality was initially said to have a specific EEG correlate posterior temporal slow activity. However, Fenton et al. ${ }^{1}$ studied the diagnosis of offender patients with this EEG abnormality and did not confirm the correlation between psychopathy and posterior temporal slowing. On the whole, the usefulness of the EEG in forensic psychiatry appears as limited as in general psychiatry, although a recent report ${ }^{2}$ suggests that the EEG in conjunction with personality assessments may be of potential value in the assessment of psychopathy.

On the other hand, the EEG is the investigation of choice in suspected cases of epilepsy, and the relationship between psychosis and epilepsy is well documented. In particular, there appears to be a relationship between temporal lobe abnormalities and mental illness.

Gruhle $^{3}$ and then $\mathrm{Hill}^{4}$ and Pond ${ }^{5}$ reported psychotic states in epileptic patients that closely resembled schizophrenia. In their reviews nearly all the patients had temporal lobe lesions. In a comprehensive study, Slater ${ }^{6}$ examined 69 epileptics pre- senting with a schizophreniform psychosis; $80 \%$ had temporal lobe epilepsy (TLE) and differences were noted between the presentation of these patients and schizophrenia in non-epileptic patients. In particular, there was an absence of pre-morbid schizoid traits and a maintenance of a good affective response and rapport. Flor-Henry ${ }^{7}$ in a study of patients with psychosis and TLE found that dominant or bilateral hemisphere foci pre-disposed to schizophreniform psychosis, but non-dominant hemisphere foci tended to pre-dispose to manic-depressive psychosis.

From the above, it can be concluded that there is an increased association between epilepsy and psychosis.

Despite the doubts and arguments about the EEG in psychiatry, EEGs are not infrequently requested by psychiatrists for their patients. With this in mind, this study was devised to examine the usefulness of the EEG, not merely as a diagnostic instrument, but as an adjunct to overall management in clinical psychiatric practice.

\section{The study}

The authors examined the records of all patients who had an EEG during the period 1 January 1984 to 31 December 1985. These patients were referred by one clinical team at Scalebor Park Hospital. An assessment was made of the referring psychiatrist's expectation, and whether the results of the EEG had significantly influenced his opinion on, or his management of, the patient involved.

The case notes of each patient were examined for 'organic' data recorded in the history of the patient's illness or in the mental or physical examination. The term 'organic' is used to refer to data concerning events which could reasonably be expected to cause, or result from, or be associated with a structural brain abnormality. 'Organic' data from the history were noted to be present if one of the following was obtained:

An episode of loss of, or impairment of, consciousness; or a confusional episode; or impairment of memory; or head injury with loss of consciousness and an associated period of amnesia; or a neurological disorder such as hemiparesis, consistent with brain abnormality.

'Organic' data were noted to be present on examination of the patient's mental state if one of the following was 
present: Disorientation for time, place or person; or memory impairment (not explicable on the basis of an attentional deficit); or aphasia, apraxia, or agnosia, or other focal neurological deficit.

'Organic' data were noted to be present on neurological examination if one of the following was observed: An abnormality of the extrapyramidal, pyramidal, sensory, or cerebellar systems (excluding drug induced syndromes); or impairment of vision or hearing (not explicable on the basis of end organ disease); or other cranial nerve abnormalities.

After these steps had been completed, the EEG results were examined and classified according to whether they were normal or abnormal. The abnormal recordings were further divided into those which were definitely abnormal and those which were equivocal.

\section{The findings}

Seventy-six patients were subjected to a total of 89 EEGs during the study period. Thirty-five were male and 41 female. The mean age of the total population was $\mathbf{4 2 . 5}$ years. Eleven patients had more than one EEG, for reasons such as equivocal first recording, drug effects on the recording and persistent organic features to the case.

The initial diagnosis of the patients and its relationship to the EEG result is presented in Table I. Of the 76 patients, 11 (14.5\%) had definitely abnormal recordings and 11 (14.5\%) had equivocal abnormal recordings. Seven patients had a history of brain insult, e.g. head injury, meningitis or subdural haematoma. Of these, five had an EEG abnormality reported.

In Table II, the EEG results are compared with the presence or absence of 'organic' data in the history or on examination. This revealed a significant association between abnormal EEG results and presence of organic data $(P<0.01)$. When equivocal abnormal results are excluded there is still a significant association, though at a reduced level of significance $(P<0.02)$.

Table III shows evidence that the referring psychiatrists could predict whether an EEG recording was going to be abnormal or not $(P<0.05)$.

Table IV shows that the EEG results influenced the clinical practice of the referring psychiatrists in 10 cases

TABLE I

Diagnosis and EEG result

\begin{tabular}{lrrr}
\hline \multicolumn{1}{c}{ DIAGNOSIS } & \multicolumn{3}{c}{ EEG RESULT } \\
\hline & \multicolumn{3}{c}{$\begin{array}{c}\text { Abnormal/ } \\
\text { Normal }\end{array}$} \\
& & Equivocal & Total \\
\hline & 2 & 6 & 8 \\
Acute organic psychosis & 6 & 3 & 9 \\
Chronic organic psychosis & 15 & 4 & 19 \\
Schizophrenia & 16 & 3 & 19 \\
Manic-depressive psychosis & 12 & 6 & 18 \\
Neurosis & 1 & 0 & 1 \\
Personality disorder & 1 & 0 & 1 \\
Drug dependence & 1 & 0 & 1 \\
Other & & & \\
\hline
\end{tabular}

TABLE II

$E E G$ recordings in relation to organic factors

\begin{tabular}{lccc}
\hline \multicolumn{4}{c}{ EEG RECORDING } \\
\hline & Normal & Abnormal & (equivocal) \\
\hline $\begin{array}{l}\text { Organic data } \\
\text { Present } \\
\text { Absent }\end{array}$ & 11 & 12 & $(5)$ \\
\hline
\end{tabular}

$P<0.01$ (chi square, $1 \mathrm{df}$ )

TABLE III

EEG recordings in relation to expectations of referring psychiatrists

EEG RECORDING

Normal Abnormal (equivocal)

\begin{tabular}{lrrr}
$\begin{array}{l}\text { Expected result: } \\
\text { Confirm }\end{array}$ & 46 & 13 & (8) \\
No organic & & & \\
$\begin{array}{l}\text { Confirm } \\
\text { Expected organic }\end{array}$ & 8 & 9 & $(3)$ \\
\hline
\end{tabular}

$P<0.05$ (chi square, $1 \mathrm{df}$ )

TABLE IV

Clinical consequences of EEG recordings

\begin{tabular}{llc}
\hline & \multicolumn{2}{c}{ EEG RECORDING } \\
\hline & Normal & Abnormal \\
\hline $\begin{array}{l}\text { Change in diagnosis } \\
\text { to functional } \\
\text { to organic }\end{array}$ & 3 & \\
$\begin{array}{l}\text { Change in treatment } \\
\begin{array}{c}\text { Change in prognosis } \\
\text { to better } \\
\text { to worse }\end{array}\end{array}$ & 0 & 0 \\
Total patients & 3 & 5 \\
\hline
\end{tabular}

(13.2\%). The three patients with a normal EEG had initial diagnoses of chronic organic psychosis (two patients) and TLE, the normal EEG led to further investigation and change of diagnosis to functional illness with accompanying change in prognosis.

One patient with an initial functional diagnosis was found to have EEG changes consistent with cerebrovascular disease. This was confirmed by further investigation and led to a change in diagnosis to chronic organic psychosis. 
The remaining six patients were all found to have temporal lobe foci and anti-convulsant medication was introduced with therapeutic effect in four cases. Further investigation of one of these patients also revealed the presence of a cerebral aneurysm.

\section{Comment}

A number of methodological flaws are apparent in the present study. Because of its retrospective nature it has not been possible to eliminate several sources of bias. For instance, the neurophysiologist reporting the EEGs was already informed of clinical details and must have made his interpretations with these in mind. This was inevitable, as the intention was to assess the significance of EEG recordings in clinical psychiatric practice.

The nature of the sample studied must also be borne in mind; the present study cannot be regarded as a random survey of psychiatric patients. They were selected by the referring psychiatrist on the grounds of his suspecting or needing to eliminate the presence of organic cerebral disorder.

The percentage of abnormal recordings warrants some comment. Non-specific abnormalities have been reported in 5-10\% of normal subjects and in up to $26 \%$ of psychoneurotic patients. Of our sample, $29 \%$ had an abnormal recording and half of these had a specific abnormality. Even the equivocal recordings demonstrated abnormality in excess of the non-specific increase in theta activity described by Williams. ${ }^{8}$

Not surprisingly there is a reliable association between abnormal EEG recordings and 'organic' factors in the history, mental state and neurological examination. Similarly, there is evidence that the psychiatrists could accurately predict what the EEG recordings would be (Table III). However, it should not be assumed that such accuracy implies that referrals were appropriate, as a large proportion of accurate predictions were of normal recordings.

The clinical value of the EEG is reflected not by the proportion of abnormal recordings but by the influence of the recordings on the patient's management, diagnosis and prognosis. Table IV shows that management, diagnosis and prognosis were influenced in $13.2 \%$ of cases. This is comparable with the usefulness of the CT scanner in psychiatry, Roberts \& Lishman $^{9}$ having found that the CT scanner influenced diagnosis, management and/or prognosis in $11.7 \%$ of patients scanned for clinical reasons.
A review of the literature by the authors revealed only one previous study that quantifies the clinical usefulness of the EEG. Colgan \& Philpott ${ }^{10}$ examined the results of 49 elderly patients referred on clinical grounds for EEG. Whilst $75.5 \%$ of recordings were abnormal, only $2 \%$ influenced future clinical practice.

We would argue that the EEG does have a place in clinical psychiatric practice, but only in selected patients. Our findings suggest that the cost-effectiveness of the EEG in psychiatry would be improved if recordings were limited to patients with 'organic' factors or in whom the referring psychiatrist anticipated an abnormal recording. However, we do not feel that the significance of these findings is high enough to exclude the usefulness of the EEG in the clinical setting when 'organic' factors and the psychiatrist's expectations are absent. Indeed, some may argue that the EEG is more useful when the abnormality is completely unexpected.

\section{References}

${ }^{1}$ Fenton, G. W., Tennent, T. G., Fenwick, P. B. C. \& RATTRAY, N. (1974) The EEG in anti-social behaviour: a study of posterior temporal slow activity in special hospital patients. Psychological Medicine, 4, 181-186.

${ }^{2}$ How ARD, R. C. (1984) The clinical EEG and personality in mentally abnormal offenders. Psychological Medicine, $14,569-580$.

${ }^{3}$ GruHLE, H. (1936) Wahn Uber den bei Epilepsie. Zeitschrift für die Gesamte, Neurologie und Psychiatrie, 154, 395-399.

${ }^{4}$ Hill, J. D. N. (1953) Psychiatric aspects of epilepsy. Medical Press, 229, 473-475.

SPOND, D. A. (1957) Psychiatric aspects of epilepsy. Journal of the Indian Medical Profession, 3, 1441-1451.

'Slater, E., Beard, A. W. \& Cuitheroe, E. (1963) The schizophrenia-like psychosis of epilepsy. British Journal of Psychiatry, 109, 95-150.

${ }^{7}$ Flor-Henry, P. (1969) Psychosis and temporal lobe epilepsy. Epilepsia, 10, 363-395.

${ }^{8}$ Williams, D. (1941) The significance of an abnormal electro-encephalogram. Journal of Neurology and Psychiatry, 4, 257-268.

${ }^{9}$ Rober TS, J. K. A. \& Lishman, W. A. (1984) The use of the CAT head scanner in clinical psychiatry. British Journal of Psychiatry, 145, 152-158.

${ }^{10}$ Colgan, J. \& Philpott, M. (1985) The routine use of investigations in elderly psychiatric patients. Age and Ageing, 14, 163-167.

A detailed list of references is available from the authors on request. 